\title{
Significados docentes sobre currículum, educación inclusiva y justicia social
}

\section{Teacher Meanings on Curriculum, Inclusive Education and Social Justice}

\author{
Claudia Inostroza-Barahona ${ }^{1}$
}

\begin{abstract}
RESUMEN
El presente artículo da cuenta de una investigación cualitativa que tuvo como objetivo conocer los significados que coordinadores/as de los Programas de Integración Escolar de colegios de enseñanza básica de la municipalidad de Santiago (Chile) tienen sobre currículum, educación inclusiva y justicia social. La investigación tuvo un carácter comprensivo e interpretativo y consistió en un estudio de caso de tipo intrínseco, que examinó la particularidad de los discursos recopilados a través de grupos focales y entrevistas en profundidad. Este examen permitió abordar los complejos procesos de implementación curricular en curso, que propician una educación inclusiva bajo el prisma de la justicia social. Al presentar este artículo se busca aportar al campo más general de la investigación educativa para la justicia social desarrollada en contextos de educación pública en Chile.
\end{abstract}

Palabras claves: currículum; educación; justicia social; segregación escolar; sistema educativo.

\begin{abstract}
This article reports on a qualitative research that aimed to understand the meanings that coordinators of the School Integration Programs (PIE) of primary schools of municipality of Santiago (Chile) have about curriculum, inclusive education and social justice. This research was comprehensive and interpretive in nature, and consisted of an intrinsic case study that examined the particularity of the discourses collected through focus groups and in-depth interviews. This examination made it possible to address the complex processes of curricular implementation underway that promote inclusive education under the prism of social justice. Presenting this research seeks to contribute to the more general field of educational research for social justice developed in public education contexts in Chile.
\end{abstract}

Keywords: Curriculum; education; social justice; school segregation; educational system.

\footnotetext{
${ }^{1}$ Docente, Escuela Benjamín Vicuña Mackenna, Santiago, Chile; Magíster en Educación, mención Currículo y Comunidad Educativa, Universidad de Chile, Santiago, Chile; clinostroza@ug.uchile.cl.
} 


\section{Introducción}

El presente artículo expone los resultados de una investigación cualitativa que tuvo por objetivo general conocer los significados que los y las docentes de Chile atribuyen al currículum, la educación inclusiva y la justicia social.

El problema que motivó la realización de esta investigación se relaciona con las desigualdades e injusticias que son validadas por el sistema educativo chileno, particularmente en el contexto de la educación pública. Los grupos socioeconómicamente favorecidos controlan las decisiones políticas y económicas, en detrimento de los grupos excluidos. Ello incrementa los niveles de injusticia social, al mismo tiempo que se intenta hacer creer que estas desigualdades no son tan amplias, legitimando así un orden mundial estratificado, tal como lo ha señalado el economista Joseph Stiglitz (2012).

De acuerdo a la OCDE, Chile es uno de los países más desiguales del mundo en términos socioeconómicos (Organización para la Cooperación y el Desarrollo Económicos [OCDE], 2018). Lo anterior se puede constatar en los resultados arrojados por encuestas aplicadas a nivel nacional, en las que se evidencia una alta desigualdad en la distribución de los ingresos (Casen, 2009).

El sistema educacional chileno no es ajeno a esta realidad y posee, por tanto, todas aquellas limitaciones propias de un sistema segmentado, producto de la desigualdad social y económica. Como lo plantea la OCDE, el sistema educacional chileno se encuentra conscientemente estratificado por clases sociales (OCDE, 2004).

En este contexto, resulta imprescindible observar, bajo el prisma de la justicia social, el poder que tiene la educación para impulsar transformaciones sociales (Murillo y HernándezCastilla, 2014). En esta línea, la educación para la justicia social constituye un nuevo enfoque teórico-práctico que busca conocer y desarrollar la consecución de una sociedad más justa. 


\section{Revisión de literatura}

Una herramienta fundamental con la que cuenta el sistema educativo es el currículum, el cual se convierte en un poderoso instrumento que posibilita ver la educación de una manera global e integral, incorporando la teoría y la práctica e imprimiendo un sello político, ideológico, axiológico y epistemológico, puesto que todo currículum refleja decisiones políticas determinadas, las cuales no son ni neutras ni arbitrarias (Magendzo, 2010). En el contexto social actual, el cual es tremendamente desigual e injusto, el currículum contribuye de forma muy eficaz a reproducir y legitimar las diferencias sociales (Jornet, 2016).

En las últimas décadas, la educación inclusiva se ha constituido en uno de los pilares fundamentales de las políticas educativas mundiales. Esta categoría surge de la confluencia de una serie de movimientos que tienen por objetivo enfrentar la exclusión, discriminación y desigualdad en las que se ven envueltos múltiples colectivos de personas (Blanco, 2006). Se trata de un conjunto de procesos que implican cambios profundos a nivel de culturas, políticas y prácticas. En los últimos años, una nueva concepción en la literatura ha postulado que la educación inclusiva tiene directa relación con la democracia, afirmando que las prácticas inclusivas deberían enmarcarse en el contexto de la justicia social (Echeita et al., 2013).

La educación inclusiva se basa en un modelo social que plantea que las barreras para el aprendizaje y la participación emergen de la interacción de los sujetos con sus contextos. Forman parte de dichos contextos las personas, políticas, instituciones y culturas, así como las condiciones sociales y económicas que afectan las vidas de los individuos. La utilización del concepto de barreras para el aprendizaje y participación representa un cambio de enfoque, que hace referencia a las dificultades que cualquier estudiante experimenta durante su vida escolar (Booth y Ainscow, 2002).

Es así como diversos autores, entre ellos Booth y Ainscow, sostienen que las escuelas inclusivas requieren del avance en tres dimensiones principales: culturas, políticas y prácticas. Este avance es entendido como un proceso de transición desde una educación segregada hacia una para la diversidad. Hoy en día la tendencia mundial es promover la plena participación de todos/as los/as estudiantes. Es imperioso pensar la educación de una forma 
inclusiva, donde se validen las diferencias y se potencien en conjunto. Al igual que en otras áreas de la sociedad, esto no ha sido nunca tan importante como hoy.

La justicia social, por su parte, se presenta como un término altamente complejo, político y cambiante. Resulta preciso destacar que en las últimas décadas se han producido una serie de acontecimientos, como la globalización, los movimientos migratorios, el aumento del desempleo y las sucesivas crisis, que han provocado un incremento en la complejidad de la sociedad y en las causas de las desigualdades e injusticias por razón de género, clase social, cultura, capacidad, orientación sexual, entre otros. Es por ello que la justicia social es un concepto dinámico que se debe comprender en relación con la sociedad en la que se enmarca. Su definición estará asociada también a la manera en que dicha sociedad comprenda la educación y su papel en la construcción de una ciudadanía democrática (Murillo y Hernández-Castilla, 2014).

Frente a las injusticias que se viven actualmente en la sociedad, la educación es uno de los procesos más relevantes para compensar dichas injusticias y promover la movilidad social. En este sentido, es preciso rescatar el concepto de justicia social en la educación, el cual últimamente ha sido reemplazado por el de "equidad educativa" (Murillo y HernándezCastilla, 2011). Para profundizar en el concepto de justicia social en educación, se apela a las dimensiones conocidas como "las tres R": redistribución, reconocimiento y representación.

La primera dimensión está centrada en la redistribución de recursos materiales y culturales. Para que exista justicia social en la educación, es primordial que todas las personas puedan tener las mismas oportunidades desde el inicio. Una educación justa se define, en primer lugar, por su capacidad para redistribuir los recursos materiales y culturales de forma tal que garantice la igualdad de oportunidades. Diversos estudios han demostrado que una mayor cantidad de años en el sistema educativo permite acceder, con mayores probabilidades de éxito, al mundo laboral, la movilidad social y la salida de la pobreza (Blanco, 2006). De este modo, una política y una institucionalidad educativa socialmente justas deben ofrecer todos los medios necesarios para compensar las desigualdades y situaciones de desventaja inicial (Bolívar, 2005). 
La segunda dimensión está relacionada con el reconocimiento y el respeto cultural de todas y cada una de las personas, y en la existencia de relaciones justas dentro de la sociedad. Desde la dimensión del reconocimiento en educación, es necesario considerar que los grupos minoritarios o tradicionalmente excluidos o marginados requieren un currículum y unos procesos de enseñanza y aprendizaje que reconozcan y valoren su cultura, sus historias particulares y sus estilos de vida (Tikly y Barrett, 2011).

Finalmente, la tercera dimensión está referida a la representación y participación de las y los estudiantes en el ámbito educativo, en todos aquellos asuntos que afectan sus propias vidas, es decir, asegurar que todas/os puedan tener una activa participación en la escuela.

Uno de los mayores retos que enfrentan los sistemas educativos alrededor del mundo, y particularmente en Chile, es responder con justicia social. En este escenario, la inclusión ha de ser concebida como una política transversal, orientada a la construcción de comunidades educativas que acojan a todos/as, valoren la diversidad y se preocupen por el bienestar y desarrollo de sus integrantes (Murillo y Duk, 2010). Todo lo anterior, cuestionando los complejos procesos de la implementación curricular y las decisiones pedagógicas, conceptuales, políticas e ideológicas que hoy perpetúan un sistema educativo desigual e injusto.

\section{Método}

La investigación cualitativa realizada se orientó a analizar casos concretos, particulares y temporales, a partir de expresiones y actividades de las personas en sus propios contextos (Flick, 2004). La investigación, además, fue de tipo intrínseco, ya que analizó en profundidad la interacción de factores que producen cambio, crecimiento o desarrollo en casos particulares seleccionados (Pérez, 2001).

La fuente de información del estudio, tal como es propio de la investigación cualitativa, son sujetos protagonistas de ciertas realidades. En este sentido, la metodología cualitativa está orientada a captar estructuras de significados con las que pueden describirse prácticas 
culturales, atendiendo las propias comprensiones de los sujetos (Canales et al., 2016). A partir de esta perspectiva, los/as informantes claves colaboran con datos ricos, diversos y profundos acerca de sus experiencias pedagógicas, en el ámbito educativo del currículum, la educación inclusiva y la justicia social.

La muestra de la presente investigación se circunscribió a coordinadoras/es de Programas de Integración Escolar (PIE), que se desempeñan en escuelas de enseñanza básica ubicadas en la municipalidad de Santiago, Chile. El Programa de Integración Escolar corresponde a una estrategia inclusiva que surge en las últimas décadas en Chile, a partir de nuevas políticas públicas que buscan valorar la diversidad. El criterio de selección de los/as informantes claves fue que correspondiesen a profesionales de la educación a cargo de la coordinación de los respectivos PIE, realizándose una selección de muestra intencionada no probabilística.

Se aplicaron entrevistas en profundidad y grupos focales, técnicas que fueron seleccionadas debido a su complementación mutua. Mientras que las entrevistas en profundidad permitieron obtener representaciones de carácter individual, los grupos focales permitieron acceder a representaciones colectivas.

Para la muestra se utilizaron criterios que sustentaron una selección que saturó el espacio simbólico a investigar, con el fin de obtener la mayor información posible y relevante. De este modo, la muestra "no responde a criterios estadísticos, sino estructurales, es decir, a determinadas relaciones sociales" (Delgado y Gutiérrez 1999, p. 298). De igual forma, la representatividad de la muestra fue definida a partir de un criterio de saturación, bajo la premisa de que toda información producida al interior de un grupo es finita, por lo que, llegado cierto punto, esta se agota y comienza a redundar, indicando que ya es tiempo de detener el proceso de producción de información (Canales, 2006).

Para conocer los significados que las/os coordinadoras/es de los Programas de Integración Escolar le atribuyen al currículum, se examinaron dos dimensiones: los procesos de implementación y flexibilización curricular. Con respecto a los procesos de implementación, se indagó en las estrategias pedagógicas utilizadas por las escuelas y, para los procesos de 
flexibilización curricular, se buscó comprender qué acciones toman los/as coordinadores/as para adaptar el currículum.

Respecto a la educación inclusiva, y para conocer los significados que se le atribuyen a esta, se recabó información sobre elementos que facilitan u obstaculizan la construcción de una cultura inclusiva. Finalmente, para conocer de manera más exhaustiva los significados que los y las coordinadores/as atribuyen a la justicia social para la educación, se buscó conocer qué acciones concretas estos/as realizan para lograr que todos/as los/as estudiantes aprendan de manera justa. Se buscó también conocer cómo se interpretan las dimensiones de la justicia social en el ámbito educativo, desde el punto de vista de los y las coordinadores/as.

Con objeto de reducir eventuales sesgos producidos en la recogida de datos, se optó por la realización de una triangulación de la información obtenida. Esta estrategia de validación consistió en la aplicación de entrevistas y grupos focales. De esta manera se permite verificar la estabilidad de los resultados producidos (Mucchielli, 2001).

\section{Resultados}

Es posible afirmar que los significados que poseen los/as coordinadores/as de Programas de Integración Escolar responde a la experiencia pedagógica y al mundo intersubjetivo propio de una sociedad marcada por la desigualdad y el modelo neoliberal predominante, que orienta y define las políticas educativas. Existe el reconocimiento de la educación como un derecho y la consideración de la diversidad para la transformación de la escuela, entendida esta como una revolución en proceso.

Los significados que los/as coordinadores/as de los Programas de Integración Escolar atribuyen al currículum, la educación inclusiva y la justicia social, tienen relación con la valoración de la diversidad y su incorporación al desarrollo curricular como un criterio de referencia esencial. Consecuentemente, puede decirse que para ellos/as una condición fundamental del currículum de un país es su "flexibilidad para propiciar procesos educativos 
diversificados" (Duk y Loren, 2016, p.188). Todo lo anterior, para la construcción de escuelas inclusivas y avanzar hacia sociedades justas y democráticas (Blanco, 2006).

En relación a los procesos de implementación del currículum, hay sustanciales diferencias entre escuelas, las que tienen que ver con dónde se reubica, se reenfoca y se recontextuliza el currículum (Magendzo, 2008). En los discursos de las/os coordinadoras/es de los Programas de Integración Escolar, es posible encontrar determinadas visiones que orientan las decisiones curriculares que sustentan la implementación del currículum, las que a su vez poseen un trasfondo político e ideológico.

Respecto al proceso de construcción de una cultura inclusiva, los discursos expresados por los/as coordinadores/as manifiestan una voluntad de cambio y transformación estructural de las instituciones escolares. Lo anterior evidencia la necesidad de un cambio en la cultura curricular respecto a la estructura del sistema escolar, en donde todos los agentes de la comunidad educativa sean protagonistas al momento de propiciar dicho cambio.

En relación a los significados que los/as coordinadores/as de los Programas de Integración Escolar le atribuyen a la categoría de justicia social en educación, es posible mencionar que estos/as reconocen intuitivamente las dimensiones de redistribución, reconocimiento y representación. En cuanto a las acciones concretas que realizan los/as coordinadores/as para lograr que todos los/as estudiantes aprendan de manera justa, se observó que estas tienen relación con velar por el cumplimiento adecuado de normativas legales que resguarden la participación y el acceso a la educación. Lo anterior se vincula a una comprensión de la justicia social para la educación, que asume que la educación debe mejorar las oportunidades de vida de los estudiantes.

\section{Discusión y conclusiones}

Los significados que los/as coordinadores/as de los Programas de Integración Escolar atribuyen al currículum, la educación inclusiva y la justicia social, dicen relación con la valoración de la diversidad y su incorporación al desarrollo curricular como un criterio de 
referencia esencial. Existe una clara intención y necesidad de avanzar hacia la generación de culturas educativas inclusivas y justas, lo cual se demuestra en las demandas de un replanteamiento amplio y sistémico de los centros educativos y la construcción de las políticas educativas. Consecuentemente, a nivel local se constata la imperiosa necesidad de adoptar un desarrollo curricular para la educación inclusiva, desde el prisma de la justicia social.

Es posible afirmar que estamos frente a una problemática en la formación inicial docente, ya que esta presenta carencias en relación a los conceptos analizados, revelándose la necesidad de cultivar un mayor entendimiento de los principios de la educación inclusiva y la justicia social. Por tanto, la tarea primordial recae en las instituciones formativas, lo cual demanda cambiar el paradigma dominante, para conseguir progresos más significativos hacia sistemas educativos más inclusivos y sociedades más justas.

En un plano directamente educativo, apostar por una educación para la justicia social es una tarea compleja, que requerirá de los más altos niveles de decisión en cada país, pues supone revisar los fines mismos de la educación. Posteriormente, requirirá ajustar los sistemas educativos para que implementen esas nuevas decisiones, las cuales suponen cambios que permitan desarrollar pedagogías que rompan con la subordinación.

Se requiere que los actores de la educación reconozcan su responsabilidad en estos cambios. El rol de las y los líderes escolares asume un especial protagonismo a la hora de promover y establecer las condiciones necesarias para desarrollar procesos que lleven a una escuela justa. Pero no cualquier liderazgo es válido. Se requiere un liderazgo comprometido con las personas, con un estilo democrático y distribuido, que busque hacer real ese sueño de una sociedad diferente. Un liderazgo que trabaje en, desde y para la justicia social, ya que trabajar en el mundo educativo conlleva la responsabilidad ética de luchar por un mundo más justo.

Como se puede apreciar en este artículo, existe una revolución pendiente para avanzar hacia una educación justa y para todos y todas. Para eso es fundamental una participación colaborativa de los miembros de la comunidad educativa. Esta revolución será posible si las 
escuelas incorporan el concepto de justicia social en sus propios proyectos institucionales y si los propios tomadores de decisiones de políticas públicas en educación establecen regulaciones legales desde el prisma de la justicia social.

\section{Referencias}

Blanco, R. (2006). La equidad y la inclusión social: uno de los desafíos de la educación la escuela hoy. Revista Iberoamericana sobre Calidad, Eficacia y Cambio en Educación, 4(3), 1-15. https://www.redalyc.org/articulo.oa?id=551/55140302

Bolívar, A. (2005). Equidad educativa y teorías de la justicia. Revista Electrónica Iberoamericana sobre Calidad, Eficacia y Cambio en Educación, 3(2), 42-69.

Canales, C. (2006). Metodologías de investigación social. $1^{\mathrm{a}}$ ed. Lom Ediciones.

Delgado, J. y Gutiérrez, J. (1999). Métodos y técnicas cualitativas de investigación en Ciencias Sociales. Síntesis. https://doi.org/10.33776/trabajo.v3i0.233

Duk, C. y Loren, C. (2010). Flexibilización del currículum para atender la diversidad. Revista Latinoamericana de Educación Inclusiva, 4(1), 187-210.

Echeita, G., \& Freire, P. (1997). Pedagogía de la autonomía. Siglo XXI.

Echeita, G., Simón, C. Sandoval, M. y Monarca, H. (2013). Cómo fomentar las redes naturales de apoyo en el marco de una escuela inclusiva: propuestas prácticas. Eduforma.

Ministerio de Desarrollo Social. (2009). Resultados Encuesta Casen Educación. http://observatorio.ministeriodesarrollosocial.gob.cl/layout/doc/casen/casen_educaci on.pdf

Flick, U. (2004). Introducción a la investigación cualitativa. Morata. 
Jornet, J. M., Sancho-Álvarez, C. y González-Such, J. (2016). El constructo Valor Social Subjetivo de la Educación: validación cruzada entre profesorado de escuela y universidad. Revista de Investigación Educativa, 34(2), 329-350. http://dx.doi.org/10.6018/rie.34.2.226131

García-Huidobro, J. (2007). Desigualdad educativa y segmentación del sistema escolar. Consideraciones a partir del caso chileno. Pensamiento Educativo, 40(1), 65-86.

Magendzo, A. (2008). Dilemas del currículum y la pedagogía: analizando la reforma curricular desde una perspectiva crítica. Lom Ediciones.

Magendzo, A. (2010). Dilemas y tensiones curriculares y pedagógicas de la educación en derechos humanos. Revista Instituto Interamericano de Derechos Humanos (IIDH), (52), 321-330.

Mucchielli, A. (2001). Diccionario de métodos cualitativos en ciencias humanas y sociales. Síntesis. Paidós.

Murillo, F. y Duk, C. (2010). Aulas, escuelas y sistemas educativos inclusivos: la necesidad de la mirada sistémica. Revista Latinoamericana de Educación Inclusiva, 5(2), 1112.

Murillo, F. y Hernández-Castilla, R. (2011). Hacia un Concepto de Justicia Social. REICE. Revista Iberoamericana sobre Calidad, Eficacia y Cambio en Educación, 9(4),7-23.

Murillo, F. y Hernández-Castilla, R. (2014). Liderando escuelas justas para la justicia social. Revista Internacional de Educación para la Justicia Social, 3(2), 13-32.

Organización para la Cooperación y el Desarrollo Económico. (2018). Estudios económicos de la OCDE, Chile: Visión general. https://www.oecd.org/economy/surveys/Chile2018-OECD-economic-sruvey-Spanish.pdf

Pérez, G. (2001) Modelos de investigación cualitativa en educación social y animación sociocultural. Narcea. 
Stiglitz, J. (2012). El precio de la desigualdad. El 1\% de la población tiene lo que el 99 necesita. Santillana Ediciones Generales.

Tikly, L., y Barrett, A. (2011). Social Justice, Capabilities and the Quality of Education in Low Income Countries. International Journal of Educational Development, 31 (1), 3-14. https://doi.org/10.1016/j.ijedudev.2010.06.001 